\title{
Erratum
}

\section{Multiplicity among peculiar A stars}

I. The Ap stars HD 8441 and HD 137909, and the Am stars HD 43478 and HD 96391

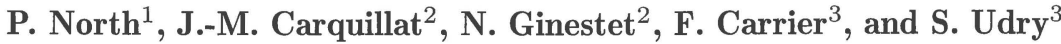

${ }^{1}$ Institut d'Astronomie de l'Université de Lausanne, CH-1290 Chavannes-des-bois, Switzerland

${ }^{2}$ Observatoire Midi Pyrénées, 14 avenue Edouard Belin, F-31400 Toulouse, France

${ }^{3}$ Observatoire de Genève, CH-1290 Sauverny, Switzerland

Astron. Astrophys. Suppl. Ser. 130 (1998) 223-232

Equation (1) should read:

$a_{2}=a-a_{1}=4.114 \pm 0.181$ U.A. $=615.210^{6} \mathrm{~km}$

The error estimate in the paper $( \pm 0.031)$ referred to the quantity $a_{1}$ instead of $a_{2}$. This was a typing mistake, so that the other error estimates (e.g. on the mass ratio) are correct.

Our thanks go to Dr. Roger Griffin for drawing our attention to this mistake. 